\title{
Effects of anterior cingulate lesions on sequential behaviors
}

\author{
N. R. REMLEY, D. C. WILSON, and G. L. SNETHERN \\ Texas Christian University, Fort Worth, Texas 76129
}

\begin{abstract}
Rats were trained on a task employing an alternating FR 4 schedule in which the subjects were required to press two bars in a left-right-left-right sequence. Lesions were placed in the anterior cingulate cortex in one group of subjects, and the other group received sham lesions. During postsurgical testing, the lesion group had significantly fewer correct sequential responses than the sham group. The ratio of number of errors to number of completed trials was significantly higher for the brain-damaged subjects, but the number of incompleted trials for the two groups did not differ.
\end{abstract}

Although a number of studies have dealt with the functional aspects of the cingulate cortex, it is still not clear what role the cingulate cortex plays in the regulation of behavior. A variety of evidence has implicated the dorsomedial cortex of the rat in the control of the sequencing of individual responses into an organized behavioral pattern (Numan, 1978; Slotnick, 1967; Thomas, Hostetter, \& Barker, 1968). Barker and Thomas (1965) trained rats on a runway task in which the animals were rewarded and nonrewarded on alternate trials. Following bilateral cingulectomy, the animals lost the ability to discriminate the sequence of events and ran consistently on both the rewarded and nonrewarded trials. In another study, a similar procedure was used by Barker and Thomas (1966). They investigated the ability of animals with either anterior or posterior cingulate cortical lesions to alternate in a runway. Posterior cingulate lesions did not produce a deficit in the performance, but there was a significant deficit in the alternation behavior of the animals having anterior cingulate damage. Although there was some recovery from the deficit, the animals with the cinguate lesions never achieved the level of performance exhibited by the control subjects. In these previous studies, the subjects had to either inhibit a response (run slower on nonrewarded trials) or exaggerate the other response (run faster on rewarded trials) to demonstrate their ability to alternate. It is possible that the subjects' lack of ability to alternately run fast and slow could be the result of the subjects' not being able to initiate an appropriate response, rather than an inability to alternate a behavioral sequence (McCleary, 1961).

The present study was designed to measure the effects of anterior cingulate lesions on alternation behavior in a two-bar operant box. The task employed an alternating FR 4 schedule in which the subjects were required to press the two bars in the sequence left-rightleft-right (LRLR) to receive a 45-mg Noyes food pellet. This task does not require the subjects to alter the mode of responding, but to simply alternately press in sequence two spatially separate bars. If the cingulate cortex plays a role in the functional organization of behavioral sequences, then animals with lesions in the cingulate cortex should be unable to maintain this sequence of responding.

\section{METHOD}

Subjects

The subjects were 12 90-day-old female Sprague-Dawley albino rats. The animals were maintained at $85 \%-90 \%$ of their free-feeding body weights during the test phases of the experiment. The animals were housed individually and were maintained on a 12-h light/dark cycle throughout the experiment.

\section{Apparatus}

Operant test chambers with two bars were used to test for the spatial alternation behavior. A white light was positioned over each bar. The presentation of stimuli, the recording of the responses, and the delivery of the reinforcements were controlled by appropriate electromechanical programming equipment housed in an adjacent room.

\section{Procedure}

The subjects were trained on a series of barpressing schedules beginning with a CRF schedule and ending with the discretetrial, LRLR, task. Each trial lasted $15 \mathrm{sec}$ and was followed by a 5 -sec intertrial interval (ITI). Each trial was begun by turning on the houselight and the cue light over the left bar. The cue light was turned off by a left barpress. The remainder of the barpressing sequence, RLR, was marked by a flash of light from the light over each bar when the bar was pressed. On each trial, the subject could do one of three things: (1) respond LRLR correctly, (2) make a sequential error by responding $L L, L R R$, $R$, or LRLL, or (3) fail to complete the correct sequence, LRLR, within the $15 \mathrm{sec}$ of the trial. Following a correct response sequence, the subject received a reinforcement, all the lights were turned out, and the subject remained in the blackout period for the remainder of that trial and the ensuing ITI. A sequential error resulted in a blackout for the remaining time of the trial and the ensuing ITI period, with no reinforcement being delivered. An incomplete response sequence resulted in a blackout during the ITI period, with no reinforcement being delivered.

The subjects were run on a schedule of 50 trials/day and to a criterion of 35 correct response sequences from the 50 trials for 3 successive days. After the subjects had reached criterion, they were put on an ad-lib schedule of feeding for 7 days. At that 
time they were given either anterior cingulectomies via aspiration or, for the sham operation, simple craniectomies. Following a 1 -week recovery period, the subjects were again food deprived to $85 \%-90 \%$ of their free-feeding weights. Upon reaching the appropriate weight level, the subjects were tested on the spatial alternation task for 5 days (250 trials). During the test phase, if any subjects failed to consistently complete a correct response sequence, it was again placed on the CRF schedule to test if the subject could perform a barpressing task and was motivated to perform by the deprivation schedule and/or the food reinforcement.

\section{RESULTS $^{1}$}

Due to excessive brain damage, one subject was omitted from the experiment, leaving five subjects in the cingulate lesion group and six subjects in the sham group. Figure 1 illustrates the minimum and maximum amount of cortical damage sustained by the subjects in the lesion group.

The data clearly reveal that animals with anterior cingulate lesions are not able to maintain an established alternation response. Figure 2 illustrates the number of correct response sequences on the alternation task during the preoperative test sessions (criterion day
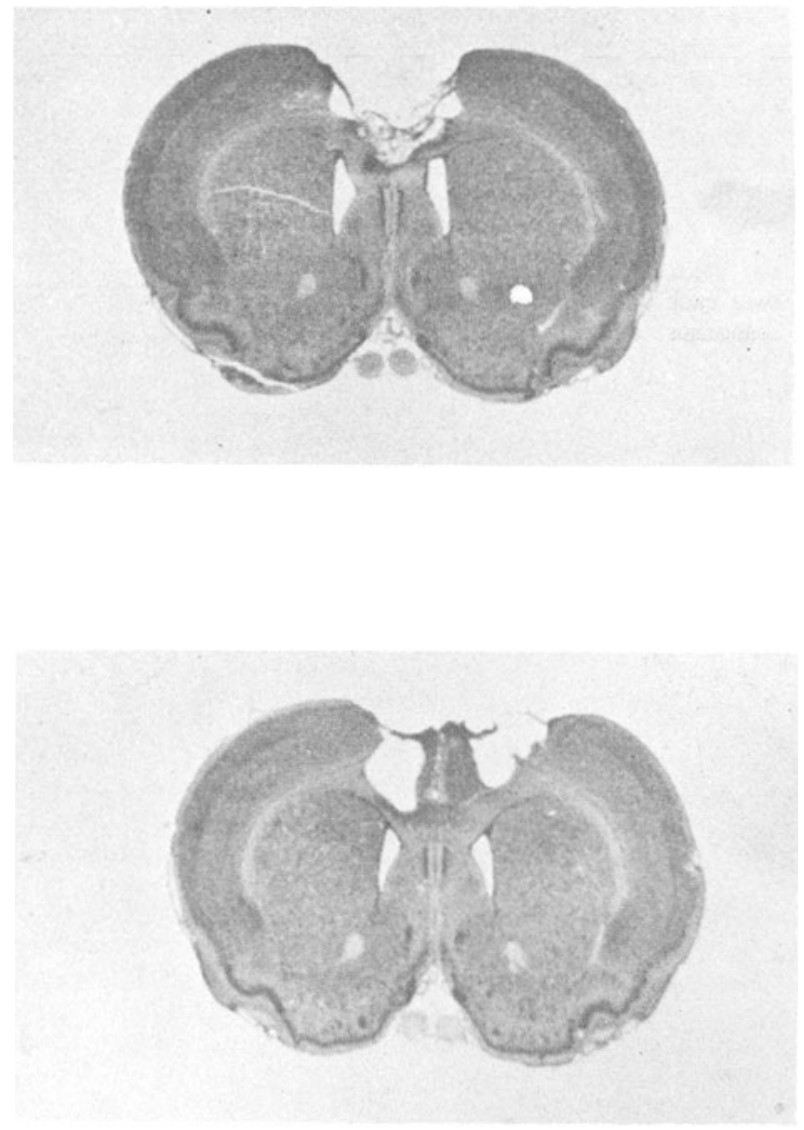

Figure 1. Examples of the amount of damage sustained by subjects with anterior cingulate lesions.

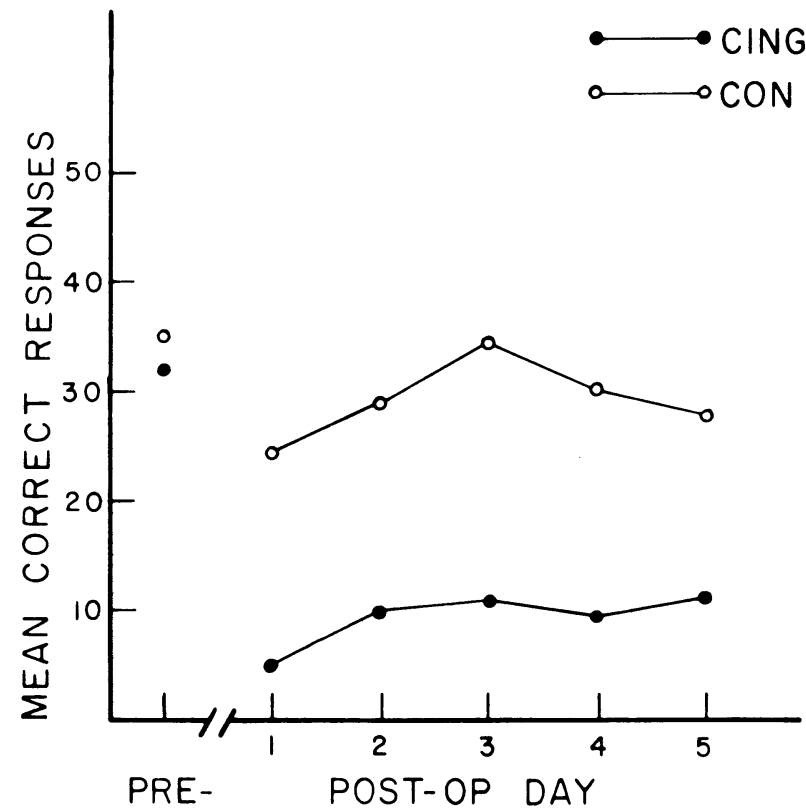

Figure 2. Mean number of correct responses for each group as a function of days. CING = anterior cingulate lesion group; $\mathrm{CON}=$ sham control group.

Table 1

\begin{tabular}{lcc}
\hline & \multicolumn{2}{c}{ Mean of 50 Trials } \\
\cline { 2 - 3 } \multicolumn{1}{c}{ Measurement } & Lesion Group & Control Group \\
\hline Correct Responses & $10.1^{*}$ & 27.7 \\
Percent Errors & $77.1^{*}$ & 42.7 \\
Incompleted Trials & $15.0 \dagger$ & 2.0 \\
\hline
\end{tabular}

${ }^{*} p<.05$. TMean for the lesion group $=8.0$ if the data of one subject are deleted.

plus two ensuing sessions) and the five postoperative test sessions. The differences between the groups on the preoperative test sessions were not statistically significant. An analysis of variance of the data for the five postoperative test sessions yielded a significant groups effect, with no significant days effect and no significant Groups by Days interaction. Separate analyses of variance were carried out on each of the following response measures: number of correct sequences, percent sequential errors (number of errors/number of total responses $X 100$ ), and the number of incompleted trials. The mean number of correct response sequences, the mean percent sequence errors, and the mean number of incompleted trials for both groups are presented in Table 1. The results of the analyses of variance indicate that the groups differed not only on the number of correct response sequences, but also on the percent of sequence errors, with the cingulate lesion group making significantly more errors than the sham group. There were no differences between the groups on the number of incompleted trials. The mean number of incompleted trials illustrated in Table 1 for the lesion group is 
abnormally inflated by the performance of one animal. If the data for this one animal are deleted from the calculation of the mean, the mean is greatly decreased. Because this animal did not consistently complete the postoperative testing sessions, it was subsequently placed on a CRF schedule of reinforcement for barpressing in a single-bar task. Its performance on this CRF schedule did not differ from its performance on the CRF task used in the preoperative training sessions, demonstrating that the subject was capable of performing a barpress task for food reinforcement.

\section{DISCUSSION}

There are two possible explanations for these data. It is possible that the animals with the cingulate lesions were not able to alternately press the two bars because of some inability to initiate responses. This is the more common explanation of failure to alternate in animals with anterior cingulate lesions (McCleary, 1966). An alternative explanation suggests that animals with anterior cingulate lesions are not able to main tain a sequential chain of responses, although the integrity of each individual response is maintained. If lesions of the anterior cingulate cortex result in an inability to intitiate responses, then we would expect that subjects with these lesions would have few, if any, complete trials. The fact that subjects with anterior cingulate lesions did not differ in the number of incomplete trials from the sham subjects suggests that the lesion group was not suffering from a deficit in response initiation. This conclusion is also supported by the fact that the one lesion subject that did not consistently complete the postoperatative testing sessions was able to respond appropriately on a single-bar FR 4 task. If the subjects were unable to inhibit a response, they would also appear to make more sequential errors. This explanation can be ruled out by the fact that subjects with anterior cingulate lesions have been shown to inhibit responses as well as or better than normals (McCleary, 1966). It therefore seems more probable that the animals with the anterior cingulate lesions were not able to maintain the chain of alternating responses because they suffered from a behavioral organization deficit rather than a deficit in response initiation. This interpretation is supported by the fact that the subjects with anterior cingulate lesions did attempt to respond but were unable to maintain the appropriate response sequence, resulting in a significantly higher percentage of sequential errors. These data do not allow us to state the exact mechanism that is responsible for the deficit in sequential behavioral organization, but they do suggest that the ability to organize behavior into meaningful patterns requires the integrity of the anterior cingulate cortex.

\section{REFERENCES}

Barker, D. J., \& Thomas, G. J. Ablation of cingulate cortex in rats impairs alternation learning and retention. Journal of Comparative and Physiological Psychology, 1965, 60, 353-359.

Barker, D. J., \& Thomas, G. J. Effects of regional ablation of midline cortex on alternation learning in rats. Physiology \& Behavior, 1966, 1, 313-317.

LuBAR, J. F., \& Numan, R. Behavioral and physiological studies of septal function and related medial cortical structures. Behavioral Biology, 1973, 8, 1-25.

Numan, R. Cortical-limbic mechanisms and response control: A theoretical review. Physiological Psychology, 1978, 6, 445-470.

McCleary, R. A. Response specificity in the behavioral effects of limbic system lesions in the cat. Journal of Comparative and Physiological Psychology, 1961, 54, 605-613.

MCCleARY, R. A. Response-modulating functions of the limbic system: Initiation and suppression. In E. Stellar \& J. M. Sprague (Eds.), Progress in physiological psychology (Vol. 1). New York: Academic Press, 1966.

Slotnick, B. M. Disturbances in maternal behavior in the rat following lesions of the cingulate cortex. Behavior, 1967, 29, 204-236.

Thomas, G. J., Hostetter, G., \& Barker, D. J. Behavioral function of the limbic system. In E. Stellar \& J. M. Sprague (Eds.), Progress in physiological psychology (Vol. 2). New York: Academic Press, 1968.

\section{NOTE}

1. The predetermined alpha level was set at $p=.05$. All results with $\mathrm{p}<.05$ are reported as being statistically significant.

(Received for publication September 21, 1981.) 